\title{
Surface modification of stereoregular and stereocomplex poly(methyl methacrylate) films with biologically identified peptides
}

\author{
Takaaki Date $^{1,4}$, Satomi Yoshino ${ }^{2}$, Hisao Matsuno ${ }^{3,5}$ and Takeshi Serizawa ${ }^{1,4}$ \\ Polymer Journal (2012) 44, 366-369; doi:10.1038/pj.2011.147; published online 18 January 2012
}

Keywords: binding; biotin-streptavidin interaction; peptide; poly(methyl methacrylate); soft interface; stereoregularity; surface modification

\section{INTRODUCTION}

With the increased use of polymeric materials in the biomedical field, surface modification techniques to create desired interfaces between artificial materials and organisms have become increasingly important in modern science and technology. ${ }^{1}$ An effective approach for developing a desired biomaterial is to modify the surface of a material that has excellent bulk properties. To modify a surface by a covalent approach often requires surface pre-treatments or harsh reaction conditions, as many polymers are chemically inert. ${ }^{2}$ Furthermore, it is inevitable that not only the chemical structures will change, but also the nanostructures of the surfaces will be destroyed. Therefore, covalent modification is sometimes not appropriate for the modification of soft and delicate materials possessing key nanostructures. On the other hand, noncovalent modification such as physical adsorption and selfassembly are very simple, ${ }^{3}$ but unfortunately depend on unpredictable and nonspecific interactions, which are difficult to control precisely.

Recently, as an alternative approach, material-binding peptides (MBPs), which specifically bind to inorganic/organic material surfaces, have been identified from combinatorial peptide libraries displayed on phages and cell surfaces, and have been used as noncovalently bound linkers to functionalize pristine surfaces. ${ }^{4}$ As it has been demonstrated that MBPs can recognize slight differences in the delicate chemical structures of material surfaces, they can be used to modify complicated material surfaces easily. This approach has been widely applied to the attachment of proteins, ${ }^{5}$ cells, ${ }^{6}$ inorganic particles $^{7}$ and polyethylene glycol $^{8}$ to target materials. Although many applications have been demonstrated for MBPs as surface modifiers, it remains an open question whether each peptide can express their capabilities to the maximum.
With regard to peptides on polymer film surfaces, the situation would be more complicated because the interfaces between the polymer film surfaces and aqueous solutions have been reported to be diffuse. ${ }^{9,10}$ In other words, the films' outermost surface of waterinsoluble polymers such as poly(methyl methacrylate) (PMMA) tends to swell with water molecules, thus forming 'soft' interfaces. Neutron reflectivity measurements have recently suggested that the thickness of the water-swollen layers of atactic (at) (in fact, syndiotactic (st)-rich) PMMA films was more than $8 \mathrm{~nm}$ from the outermost surface. ${ }^{9}$ It has also been suggested that the water-swollen layers of isotactic (it)PMMA might be thicker than that of atactic-PMMA. ${ }^{10}$ Therefore, considering the functional modification of PMMA surfaces using short peptides (for example, the length of 10-mer peptides with an extended chain conformation is expected to be less than $4 \mathrm{~nm}$ ), the peptides on the PMMA film surfaces might be embedded inside the water-swollen layers, and would not function as surface modifiers (Figure 1, top). In contrast, when the polymer film surfaces have unswollen, 'hard' interfaces, then immobilized peptides would work successfully (Figure 1, bottom). Although there has been no research on these effects on the surface modification thus far, it can be one of the important factors, which are unique to polymer surface modification using MBPs.

In this paper, we modified it- and st-PMMA film surfaces with biotinylated peptides that specifically bound to the polymers, ${ }^{11,12}$ and analyzed the binding of streptavidin (SAv) to these immobilized peptides. In addition, PMMA stereocomplex (SC) films, which were composed of both it- and st-PMMAs, ${ }^{13}$ were similarly evaluated by their specific peptides under the same conditions, and the results were compared against those of it- and st-PMMAs. We report herein that

${ }^{1}$ Research Center for Advanced Science and Technology (RCAST), The University of Tokyo, Meguro-ku, Tokyo, Japan; ${ }^{2}$ Graduate School of Engineering, The University of Tokyo, Bunkyo-ku, Tokyo, Japan and ${ }^{3}$ Komaba Open Laboratory (KOL) The University of Tokyo, Komaba, Meguro-ku, Tokyo, Japan

${ }^{4}$ Current address: Department of Organic and Polymeric Materials, Tokyo Institute of Technology, 2-12-1 O-okayama, Meguro-ku, Tokyo 152-8552, Japan

${ }^{5}$ Current address: Department of Applied Chemistry, Kyushu University, 744 Motooka, Nishi-ku, Fukuoka 819-0395, Japan

Correspondence: Professor T Serizawa, Research Center for Advanced Science and Technology (RCAST), The University of Tokyo, 4-6-1 Komaba, Meguro-ku, Tokyo 153-8904, Japan.

E-mail: t-serizawa@bionano.rcast.u-tokyo.ac.jp

Received 27 October 2011; revised 5 December 2011; accepted 9 December 2011; published online 18 January 2012 
the ability to functionally modify the film surfaces with the peptides differs depending on the PMMA species.

\section{EXPERIMENTAL PROCEDURE}

\section{Materials}

It-PMMA (Polymer Source, $M_{\mathrm{n}} 19000, M_{\mathrm{w}} / M_{\mathrm{n}} 1.10, \mathrm{~mm} 98 \%$ ), st-PMMA (Polymer Source, $M_{\mathrm{n}} 18600, M_{\mathrm{w}} / M_{\mathrm{n}} 1.23, \mathrm{~mm}>85 \%$ ), and SAv (Thermo Fisher Scientific, Waltham, MA, USA) were used as purchased. It- and st-PMMA films were prepared by spin-coating from chloroform solutions $\left(1.7 \mathrm{mg} \mathrm{m}^{-1}\right)$ onto gold-coated glass slides (SIA Kit Au, GE Healthcare, Uppsala, Sweden). SC films were prepared by the layer-by-layer assembly method, ${ }^{14}$ which is the simple alternating immersion of gold-coated glass slides into acetonitrile solutions $\left(1.7 \mathrm{mg} \mathrm{ml}^{-1}\right)$ of it- and st-PMMAs. ${ }^{13}$ The films thicknesses were adjusted to approximately $10 \mathrm{~nm}$ (see the Supplementary Information for the detailed experimental methods). Biotinylated peptides were prepared by solid-phase peptide synthesis using standard 9-fluorenylmethyloxycarbonyl -based procedures and using fluorenylmethyloxycarbonyl-glutamic acid $\gamma$-biotinylated through a mono(propylene glycol) and di(ethylene glycol) spacer as a biotinylating reagent (see the Supplementary Information for the detailed experimental methods)

\section{Film characterization}

The static contact angle of PMMA films on gold-coated glass slides (SIA Kit Au, GE Healthcare) was measured with a commercial apparatus (CA-X, Kyowa Interface Science, Saitama, Japan). The surface topology of the PMMA films was visualized by non-contact mode atomic force microscopy (AFM) (SPM9600, Shimadzu, Kyoto, Japan) in air using a silicon cantilever (PointProbe, $\mathrm{NCH}$, resonance frequency $320 \mathrm{kHz}$, force constant $42 \mathrm{Nm}^{-1}$, NanoWorld, Neuchatel, Switzerland). Film thickness of the PMMA films was measured by scratching-mode AFM (SPM-9600, Shimadzu) in HBS-N (10 mM HEPES buffer containing $150 \mathrm{~mm} \mathrm{NaCl}$, pH 7.4, GE Healthcare) using a silicon nitride cantilever (OMCL-TR800PSA-1, spring constant $0.57 \mathrm{Nm}^{-1}$, Olympus, Tokyo, Japan). The density of PMMA films was calculated from the film thickness and the film weight measured by using quartz crystal microbalance (see the Supplementary Information for the detailed experimental methods).
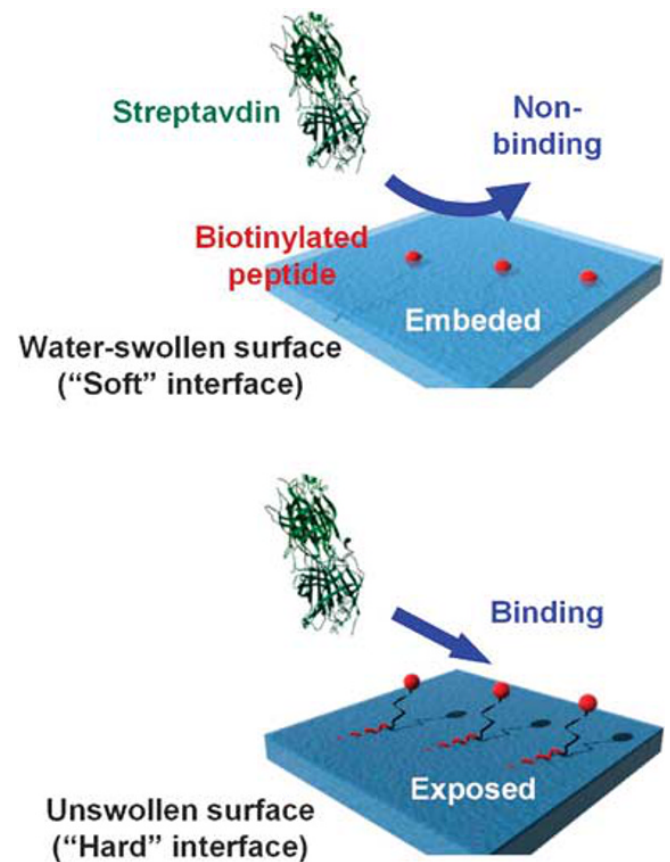

Figure 1 Schematic representation of the polymer surface modification with peptides. The peptides are embedded inside (top) and exposed on (bottom) the polymer films. Streptavidin binds to the exposed biotinylated peptides.

\section{Surface modification of PMMA films with peptides}

Surface plasmon resonance (SPR) measurements (BIAcore X, GE Healthcare) were used for surface modification experiments. PMMA films prepared on gold-coated glass slides were set to the SPR apparatus. HBS-N was flowed at a rate of $5 \mu \mathrm{min}^{-1}$ at $25^{\circ} \mathrm{C}$ during the experiment. After more than $3 \mathrm{~h}$ of buffer flow, freshly prepared biotinylated peptide solutions were applied to the PMMA films to immobilize the biotinylated peptides, and then the solutions were exchanged to a peptide-free buffer for $5 \mathrm{~min}$. Subsequently, the SAv solution ( $250 \mathrm{~nm})$ was applied for 2 min to monitor the binding of SAv, and then the SAv solution was replaced by buffer. The amount of SAv bound was calculated as the difference in the RU (resonance unit) between the baseline and $120 \mathrm{~s}$ after buffer exchange. The RU value was converted to a peptide density (pmol cm${ }^{-2}$ ), assuming that a SPR angle shift of $1^{\circ}(=10000 \mathrm{RU})$ corresponds to $\sim 1 \mu \mathrm{g} \mathrm{cm}^{-2}$. . $^{2}$

\section{RESULTS AND DISCUSSION}

Combinations of PMMAs and the peptides used for surface modification experiments are summarized in Table 1. It-PMMA-binding $(\text { itBP })^{11}$ and st-PMMA-binding peptides $(\mathrm{stBP})^{12}$ were identified previously. To demonstrate the functions of itBP and stBP as surface modifiers, the peptides were biotinylated (Figure 2a, termed B-itBP and stBP-B, respectively) for $\mathrm{SAv}$ immobilization onto the corresponding polymer films. The $\mathrm{N}$ terminus of itBP and the $\mathrm{C}$ terminus of stBP were biotinylated, because a peptide terminus which is far from the binding motif was reported to be more suitable for conjugating a foreign molecule to maintain the original affinity of the peptide. ${ }^{16,17}$ The SC-binding peptide (SCBP) was newly identified by the same experimental method (see the Supplementary Information for the detailed experimental methods). The binding constant of the SCBP for the SC films was estimated to be $7.5 \times 10^{5} \mathrm{M}^{-1}$, which was comparable with that of itBP $\left(2.8 \times 10^{5} \mathrm{M}^{-1}\right)$ and stBP $\left(9.1 \times 10^{4} \mathrm{M}^{-1}\right) .{ }^{18}$ The binding specificity of SCBP for the SC films was demonstrated (see the Supplementary Information for the detailed Results and discussion). The sequence homologies of the SCBP with other identified peptides for the SC films suggested that the amino acid sequence near the $\mathrm{C}$ terminus was important for binding with SC. Therefore, the $\mathrm{N}$ terminus of SCBP was biotinylated (Figure 2a, termed B-SCBP). Although the binding constants of these peptides for each PMMA film are slightly different, the amounts of peptides dissociated during SAv binding experiments could be ignored (less than 1 and $5 \%$ of the immobilized peptides were dissociated from it-/st-PMMA and SC films, respectively) due to sufficiently small dissociation rates.

Figure $2 \mathrm{~b}$ shows typical examples of SPR sensorgrams obtained when SAv was bound onto the peptide-immobilized PMMA films. Surprisingly, the amounts of SAv bound onto the it- and st-PMMA films were analyzed to be 0.11 and $0.32 \mathrm{pmol} \mathrm{cm}^{-2}$ at an optimal peptide density of $56.2 \mathrm{pmol} \mathrm{cm}^{-2}$ and $11.1 \mathrm{pmol} \mathrm{cm}^{-2}$, respectively. These values are considerably lower than the previously reported

Table 1 Combinations of PMMAs and peptides used for surface modification experiments

\begin{tabular}{lccc}
\hline Polymer & Peptide & Sequence & Biotinylation $^{\text {a }}$ \\
\hline it-PMMA & itBP & ELWRPTR $^{\mathrm{b}}$ & $\mathrm{N}$ \\
st-PMMA & StBP & HKPDANR $^{\mathrm{C}}$ & $\mathrm{C}$ \\
SC & SCBP & STPPRLW & $\mathrm{N}$ \\
\hline
\end{tabular}

Abbreviations: it, isotactic; itBP, it-PMMA-binding peptide; PMMA, poly(methyl methacrylate); SC, stereocomplex; SCBP, SC-binding peptide; st, syndiotactic; stBP, st-PMMA-binding peptide. aBiotinylated termini of peptide.

beference 11 .

'Reference 12

${ }^{\mathrm{d}}$ Ree the $\mathrm{S}$ Supplementary Information. 
a

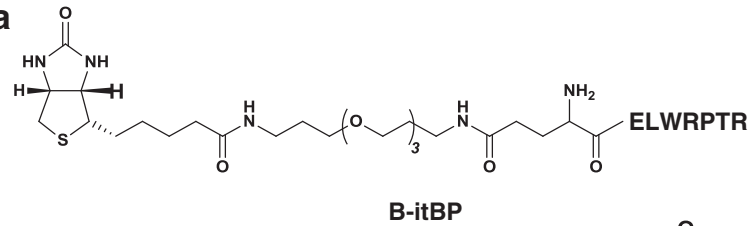<smiles>NC(=O)C(CCC(=O)NCCCOCCCNC(=O)CCCCC1SCC2NC(=O)N[C@H]21)NNCPNCc1ccccc1</smiles>

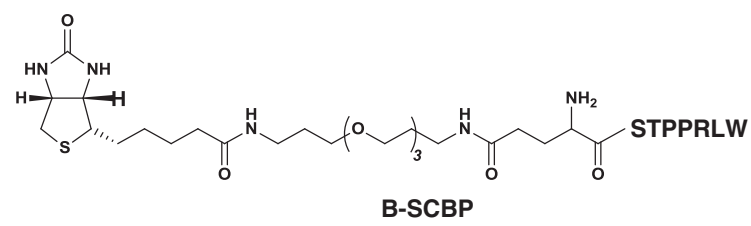

b

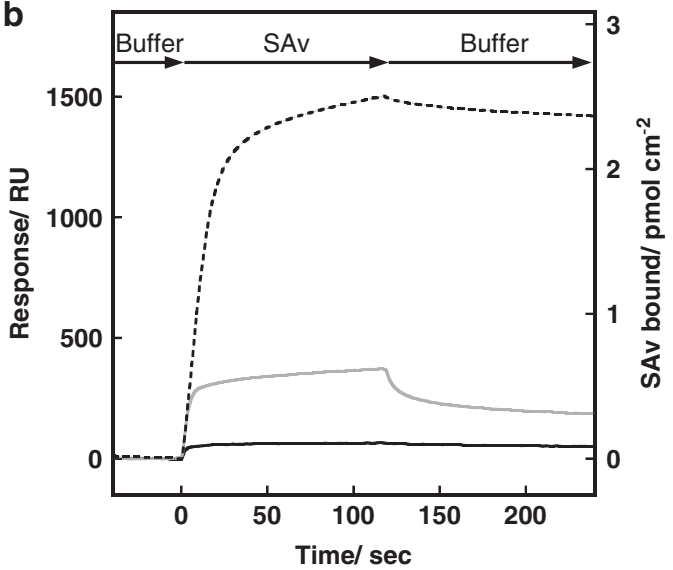

C

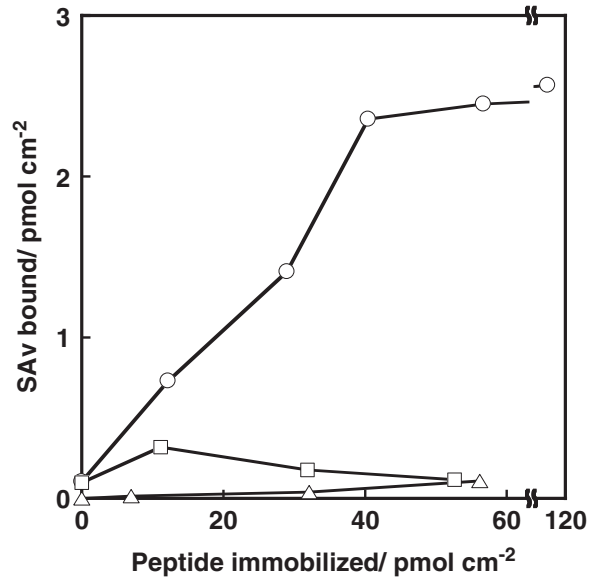

Figure 2 Surface modification of various PMMA films using biotinylated peptides. (a) Chemical structures of B-it-BP, stBP-B and B-SCBP. (b) Typical SPR sensorgrams for the binding of SAv $(250 \mathrm{~nm})$ onto peptide-coated PMMA films. Solid black line: it-PMMA/B-itBP $\left(56.2 \mathrm{pmol} \mathrm{cm}^{-2}\right)$. Solid gray line: st-PMMA/stBP-B $(11.1 \mathrm{pmol} \mathrm{cm}-2)$. Dashed black line: SC/B-SCBP $\left(56.7 \mathrm{pmol} \mathrm{cm}^{-2}\right)$. (c) The amount of SAv bound onto it-PMMA (triangle), st-PMMA (square), and SC (circle) films at various peptide densities. maximum amount of $\mathrm{SAv}\left(2.1 \mathrm{pmol} \mathrm{cm}^{-2}\right)$ bound onto polyetherimide (PEI) via the specific binding peptide under the same conditions, ${ }^{17}$ although the existence of B-itBP on it-PMMA films was confirmed by X-ray photoelectron spectroscopy (Supplementary Table S3). It is noted that the amount of SAv physically absorbed onto bare it- and st-PMMA films was similarly low (Supplementary Figure S7). When the experiments were performed at various peptide densities, the amount of SAv bound onto the it- and st-PMMA films remained low, and did not increase (Figure 2c). It was therefore difficult to simply interpret as the inaccessibility of SAv to the immobilized peptides due to steric or directional effects. It is also noted that the mean roughness values of all PMMA films were small enough (less than $2 \mathrm{~nm}$ ); therefore, the films' surface topology should not affect the bindings of peptides and SAv.

Since the molecular size of the fully extended peptides used here is less than $5 \mathrm{~nm}$, the binding of SAv seemed to be critically affected by the degree of the peptides' exposure at the polymer film surface. Most of the peptides immobilized onto the it- and st-PMMA films might be embedded inside the water-swollen layers of film surfaces, thus resulting in low accessibility of the SAv toward the peptides. Moreover, as it-PMMA films have been reported to swell with water molecules more than st-PMMA films, ${ }^{10}$ it must be reasonable that the amount of SAv bound onto the it-PMMA films was slightly less than the stPMMA films, as aforementioned. The static contact angles of the films measured in water using air bubbles also supported the embedding of the peptides inside the it- and st-PMMA films. The angles of the itPMMA films before and after B-itBP immobilization (1 mM, $30 \mathrm{~min}$ ) were estimated to be $118.6 \pm 0.7^{\circ}$ and $118.4 \pm 0.4^{\circ}$, respectively. Those of the st-PMMA films were $115.0 \pm 1.4^{\circ}$ and $115.4 \pm 0.4^{\circ}$, respectively. Therefore, the CAs for both PMMA films remained constant, implying that the surface-immobilized B-itBP and stBP-B are really embedded inside the water-swollen layers, as shown in Figure 1, top. It is noted that the PEI film's surface was more hydrophobic than the it- and st-PMMAs, possibly suggesting that the bound peptides were readily exposed at the PEI film's surface, which had a hard interface. ${ }^{17}$

On the other hand, the amount of SAv bound onto the SC films via biotinylated SCBP was significantly greater than the amount of SAv bound onto it-/st-PMMA films via B-itBP/stBP-B (Figure 2b). The amount of $S A v$ bound increased gradually with increasing density of the B-SCBP, and saturated at $\sim 2.5 \mathrm{pmol} \mathrm{cm}{ }^{-2}$ at a peptide density of more than $\sim 40 \mathrm{pmol} \mathrm{cm}^{-2}$ (Figure 2c). The amounts of SAv bound onto the SC films were comparable to those onto PEI films, irrespective of the films' hydrophilicity (see below). ${ }^{17}$ The immobilized SAv molecules were distributed as monolayer, as confirmed by AFM (Supplementary Figure S8). Although the chemical structures of these three PMMA films are essentially the same, the accessibility of the SAv toward the immobilized peptides was different, clearly indicating that these peptides on the corresponding polymer films were presented in a different way. The static contact angles of the SC films before and after B-SCBP immobilization ( $1 \mathrm{~mm}, 30 \mathrm{~min})$ were analyzed to be $125.4 \pm 0.8^{\circ}$ and $126.9 \pm 0.5^{\circ}$, respectively. In contrast to the it- and st-PMMA films aforementioned, the angles of the SC films slightly increased and became more hydrophilic after B-SCBP immobilization, possibly implying that the surface-immobilized B-SCBP might be exposed on the SC film surfaces. It is noted that the SC films were most hydrophilic in the three PMMA films based on static contact angle measurements. Therefore, it was found that the surface exposure of immobilized peptides was not simply interpreted by the contact angles of polymer films. 
A recent study on high-resolution AFM observations has proposed that SC is composed of triple-stranded helical SCs, in which it-PMMA double helices are surrounded by a single st-PMMA helix. ${ }^{19}$ Although we do not have sufficient information on the surface water-swollen structures of SC films in aqueous solution, the structurally rigid SC film surfaces might swell with fewer water molecules than the it-/st-PMMAs, thus resulting in more effective exposure of the BSCBP immobilized on the 'hard' SC film surface, as shown in Figure 1, bottom. In fact, the density of the SC films analyzed in buffer solutions was estimated to be $1.37 \mathrm{~g} \mathrm{~cm}^{-3}$, which was obviously larger than that of the it-PMMA films $\left(1.21 \mathrm{~g} \mathrm{~cm}^{-3}\right)$. Although the difference in the film densities do not directly indicate the difference in the waterswollen structures, these observations suggest that the SC films tend to swell with smaller amounts of water molecules than the it-PMMA films, followed by improvement of the peptides' ability to functionally modify the polymer film surfaces.

\section{CONCLUSION}

Biologically identified peptides that bind to it-PMMA, st-PMMA, and their assembled SC films were used for the noncovalent surface modification of polymer film surfaces, and we then compared their ability to function as surface modifiers with each other. itBP, stBP and SCBP were biotinylated, and were used for SAv immobilization onto the respective target polymers under the same conditions, thus enabling an evaluation of the accessibility of SAv toward the immobilized peptides. The SAv was almost inaccessible to the B-itBP/stBP-B immobilized onto the it-/ st-PMMA films, possibly because the peptides were embedded inside the water-swollen layers of the PMMA films. In contrast, the SAv successfully bound to the immobilized B-SCBP due to surface exposure of the peptide. Although we need to analyze the detailed structure of the SC films in the near future, it was suggested that the water-swollen structure of the polymer film surface in aqueous solution would be one of the important factors that affected their ability to functionally modify the polymer film surfaces. The present study offered new and significant insights into the modification of polymer films using MBPs, and would expand the development of MBPs for use in various applications.

\section{ACKNOWLEDGEMENTS}

We thank Professor H Aburatani (The University of Tokyo) for DNA sequencings, Professor M Komiyama (The University of Tokyo) for MALDI TOF-MS measurements and Professor M Hashizume (Tokyo University of Science) for XPS measurements. TD is grateful to the Japan Society for the Promotion of Science (JSPS) for a Research Fellow Ship for
Young Scientists. This study was financially supported in part by the Ministry of Education, Culture, Sports, Science and Technology of Japan (Grants-in-Aid for New Academic Field Research no. 21106506 to TS, and Global COE program 'Chemistry Innovation through Cooperation of Science and Engineering')

1 Castner, D. G. \& Ratner, B. D. Biomedical surface science: foundations to frontiers. Surf. Sci. 500, 28-60 (2002).

2 Kasemo, B. Biological surface science. Surf. Sci. 500, 656-677 (2002).

3 Prime, K. L. \& Whitesides, G. M. Self-assembled organic monolayers-model systems for studying adsorption of proteins at surfaces. Science 252, 1164-1167 (1991).

4 Sarikaya, M., Tamerler, C., Jen, A. K. Y., Schulten, K. \& Baneyx, F. Molecular biomimetics: nanotechnology through biology. Nat. Mater. 2, 577-585 (2003).

5 Sano, K., Ajima, K., Iwahori, K., Yudasaka, M., Iijima, S., Yamashita, I. \& Shiba, K. Endowing a ferritin-like cage protein with high affinity and selectivity for certain inorganic materials. Small 1, 826-832 (2005).

6 Sanghvi, A. B., Miller, K. P. H., Belcher, A. M. \& Schmidt, C. E. Biomaterials functionalization using a novel peptide that selectively binds to a conducting polymer. Nat. Mater. 4, 496-502 (2005).

7 Tamerler, C., Duman, M., Oren, E. E., Gungormus, M., Xiong, X., Kacar, T., Parviz, B. A. $\&$ Sarikaya, M. Materials specificity and directed assembly of a gold-binding peptide. Small 2, 1372-1378 (2006).

8 Khoo, X., Hamilton, P., O'Toole, G. A., Snyder, B. D., Kenan, D. J. \& Grinstaff, M. W. Directed assembly of PEGylated-peptide coatings for infection-resistant titanium metal. J. Am. Chem. Soc. 131, 10992-10997 (2009)

9 Tanaka, K., Fujii, Y., Atarashi, H., Akabori, K., Hino, M. \& Nagamura, T. Nonsolvents cause swelling at the interface with poly(methyl methacrylate) films. Langmuir 24, 296-301 (2008)

10 Date, T., Ishikawa, M., Hori, K., Tanaka, K., Nagamura, T., Iwahashi, M. \& Serizawa, T. Water droplets for the symmetric adhesion of two poly(methyl methacrylate) films. Chem. Lett. 38, 660-661 (2009).

11 Serizawa, T., Sawada, T., Matsuno, H., Matsubara, T. \& Sato, T. A peptide motif recognizing a polymer stereoregularity. J. Am. Chem. Soc. 127, 13780-13781 (2005).

12 Serizawa, T., Sawada, T. \& Kitayama, T. Peptide motifs that recognize differences in polymer-film surfaces. Angew. Chem. Int. Ed. 46, 723-726 (2007).

13 Serizawa, T. Hamada, K., Kitayama, T., Fujimoto, N., Hatada, K. \& Akashi, M. Stepwise stereocomplex assembly of stereoregular poly(methyl methacrylate)s on a substrate. J. Am. Chem. Soc. 122, 1891-1899 (2000).

14 Decher, G. Fuzzy nanoassemblies: toward layered polymeric multicomposites. Science 277, 1232-1237 (1997).

15 Stenberg, E., Persson, B., Roos, H. \& Urbaniczky, C. Quantitative-determination of surface concentration of protein with surface-plasmon resonance using radiolabeled proteins. J. Colloid Interface Sci. 143, 513-526 (1991).

16 Date, T., Tanaka, K., Nagamura, T. \& Serizawa, T. Directional affinity of short peptides for synthetic polymers. Chem. Mater. 20, 4536-4538 (2008).

17 Date, T., Sekine, J., Matsuno, H. \& Serizawa, T. Polymer-binding peptides for the noncovalent modification of polymer surfaces: effects of peptide density on the subsequent immobilization of functional proteins. ACS Appl. Mater. Interfaces 3, 351-359 (2011).

18 Serizawa, T., Sawada, T. \& Matsuno, H. Highly specific affinities of short peptides against synthetic polymers. Langmuir 23, 11127-11133 (2007).

19 Kumaki, J., Kawauchi, T., Okoshi, K., Kusanagi, H. \& Yashima, E. Supramolecular helical structure of the stereocomplex composed of complementary isotactic and syndiotactic poly(methyl methacrylate)s as revealed by atomic force microscopy. Angew. Chem. Int. Ed. 46, 5348-5351 (2007).

Supplementary Information accompanies the paper on Polymer Journal website (http://www.nature.com/pj) 